\title{
Assensus
}

Revista Assensus Vol 2, Núm 3

Septiembre - Diciembre 2017

ISSN 2619-3884

Recepción: Octubre 05 de 2016

Aceptación: Marzo 05 de 2017

Publicación: Septiembre 01 de 2017

\section{Estrategias pedagógicas para fortalecer los procesos etnoeducativos afrocolombianos desde la escuela.}

Pedagogical strategies to strengthen AfroColombian ethno-educational processes from school.

José de las Mercedes Mora Madera

Universidad de Córdoba - Colombia

jmoramadera@hotmail.com

Luisa Margarita Sánchez Arroyave

Universidad de Córdoba - Colombia

lujuan2000@gmail.com 


\section{Resumen}

Este artículo da cuenta de algunos avances logrados en el desarrollo del proyecto Construcción de estrategias pedagógicas para fortalecer los procesos etnoeducativos afrocolombianos desde la escuela, que se realizó desde la Maestría en Educación SUE Caribe-Universidad de Córdoba, con la comunidad educativa de la escuela El Planchón, en el municipio de Puerto Escondido, zona costanera del Departamento de Córdoba, con el propósito de fortalecer la identidad cultural a través de la intervención y transformación de los procesos educativos tradicionales, como alternativa para mejorar la calidad educativa. El estudio es de tipo cualitativo con método de investigación-acción participativa. Comprende cuatro fases: planteamiento del problema y objetivo, plan de acción, propuesta de mejoramiento y evaluación de resultados de las acciones. Los resultados muestran un grado importante de reflexión y sensibilización de los actores frente al problema, la organización del grupo IAP, diseño y aplicación de entrevistas a estudiantes, docentes, padres de familia y comunidades afro.

\section{Abstract}

This article shows some progress in the development of the construction project pedagogical strategies to strengthen Afro-Colombian ethnic education processes, conducted from the Master of Education SUE Caribbean-University of Córdoba, with the educational community of the school's Planchón in the municipality of Puerto Escondido, coastal area of the Department of Córdoba, with the purpose of strengthening cultural identity through intervention and transformation of traditional educational processes as an alternative to improve educational quality. The study is qualitative method with participatory action research. It comprises four stages: problem statement and objective action plan proposed improvement and evaluation of results of actions. The results shows a significant degree of awareness of the actors address the problem, IAP group organization, design and implementation of interviews with students, teachers, parents and African communities.

\section{Palabras Clave}

Etnoeducación, pedagógicas, currículo estrategias

Ethno-education, pedagogical strategies, curriculum 


\section{Introducción}

La educación está en constante cambio y los pedagogos tratan de adaptarse a los retos que estos cambios suponen. Por ello, día a día intentan estudiar y solucionar los problemas que en ella se presentan, creando nuevas estrategias pedagógicas, que sean pertinentes con el contexto de los estudiantes y eliminen las dificultades presentes en las aulas, ayudando así al mejoramiento de los procesos de enseñanza aprendizaje, en especial garantizar la formación integral de los niños en Colombia.

En este contexto, se tienen presente algunas consideraciones metodológicas y pedagógicas que contribuyen desde el análisis del currículo a la búsqueda de alternativas en aras de mejorar los procesos de articulación y contextualización de los saberes a partir de la exploración de la realidad sociocultural del municipio de Puerto Escondido y, en especial, de la Institución Educativa el Planchón.

En la actualidad, en muchas instituciones del Caribe colombiano, prevalece un interés para que el estudiante construya su propio conocimiento a través de la experiencia y los saberes previos, como también la necesidad de promover los procesos de crecimiento personal de los alumnos en el marco de la cultura del grupo al que pertenecen. Para el caso de esta investigación, el contexto macro corresponde a la Región costanera de Córdoba (Colombia), considerada como un espacio de cultura, de tradiciones, de vivencias, las cuales le dan identidad a la misma. Darle vida a estas tradiciones y rescatar algunos valores culturales, demanda mejorar los procesos de enseñanza y aprendizaje. Para que esto sea posible, se necesita trabajar desde las instituciones educativas con el fin de preservar y cultivar la propia cultura. De manera que la recuperación de las raíces de la etnia afrocolombiana, debe ser una de las metas institucionales en los procesos de enseñanza en las instituciones educativas, y en particular en la Institución Educativa el Planchón. Así pues, se debe tener presente que la afrocolombianidad "es el pasado ancestral, elemento clave de su identidad étnica; el cual se fortalece a través del Sistema Educativo, la familia y medios de comunicación" (Córdoba, 2008:13).

De otra parte, una de las grandes preocupaciones que tienen algunos grupos sociales y en especial la población afrodescendiente; es darle sentido de vida a muchas tradiciones, y rescatar algunos valores culturales, para mejorar los procesos de enseñanza y aprendizaje, estableciéndose así la necesidad de trabajar desde la educación con actividades y procesos articulados a esta realidad. Sin embargo, los procesos educativos que se implementan en el Municipio de Puerto Escondido, no tienen presente las características culturales propias de una población típica afrodescendiente, lo cual se evidencia al observar y caracterizar los procesos que adelantan las instituciones educativas, que han interactuado por más de una década en esta región. Los diagnósticos desarrollados permiten inferir que la mayor parte de los proyectos educativos institucionales desarrollan su trabajo a espaldas de la realidad sociocultural. A 
pesar de que los docentes, los estudiantes y la comunidad han participado en la elaboración de algunos estudios, la etnoeducación no se ve reflejada en los procesos de enseñanza y aprendizaje.

Al compartir estas ideas en la comunidad de la Institución Educativa el Planchón, algunos docentes coinciden en la necesidad de buscar un cambio en el sentido y la práctica de la educación que se imparte, a tal punto que desde el año 2010, las comunidades afrodescendientes organizadas han solicitado que los jóvenes estudiantes sean reconocidos como tales, planteándose un interés colectivo por impulsar este modelo pedagógico para el fortalecimiento de los valores culturales y, ante todo, contextualizar los contenidos y experiencias en la búsqueda de soluciones a los problemas identificados en la comunidad.

Otro aspecto que permite exponer el problema de investigación en el cual se centró este estudio, ha sido que los docentes reconocen que los alumnos presentan dificultades para relacionar los conocimientos con su cotidianidad y con el contexto escolar, para plantear preguntas y buscar respuestas a los fenómenos cotidianos de su entorno, es decir, no se contextualizan los conocimientos del ambiente pedagógico con los hechos o situaciones específicas de la vida diaria, ni confrontan sus preconceptos y teorías trabajadas en el aula de clases, con la realidad.

Al entrar en contacto con la comunidad educativa en los procesos de evaluación institucional iniciados desde enero de 2012, una de las preguntas que se ha planteado en la organización y puesta en marcha del Proyecto Educativo Institucional gira en torno a consolidar un objetivo de fondo de este proyecto de investigación, a partir de la siguiente pregunta de investigación: ¿Qué estrategias pedagógicas se pueden implementar desde el currículo para fomentar los procesos etnoeducativos afrocolombianos en la Institución Educativa El Planchón? Ese interrogante, permite direccionar los procesos de integración y participación de los estudiantes, padres de familia, comunidades organizadas con el logro de los propósitos de la institución. De ahí se desprende la necesidad de adelantar un proceso de investigación que pretenda buscar alternativas de solución de una manera participativa por parte la comunidad, entendiendo que la educación en los grupos étnicos corresponde a procesos endógenos de formación y socialización, de acuerdo con las características culturales, sociopolíticas, económicas y lingüísticas propias. Para ello, se ha avanzado en el entendimiento y análisis del tema en cuestión mediante un proceso de revisión de literatura correspondiente a diferentes ámbitos geográficos.

Desde esta perspectiva, el trabajo de investigación está dividido en diferentes Fases que muestran los alcances y logrados. La Fase I, facilitó la planificación y organización de la propuesta, realizando la conformación de un grupo que se dedicó a identificar y priorizar el interés colectivo para mejorar los procesos educativos, partir de allí se concertaron, los objetivos del estudio, los fundamentos metodológicos y teóricos. En la Fase 2, se analizaron las prácticas educativas, se hizo una caracterización y comprensión de los procesos que desarrollan los docentes en el manejo de la etnoeducación, es decir, la forma en que se asume desde el aula la diversidad étnica y cultural. 
En la Fase 3, se pudo describir y caracterizar las particularidades del modelo curricular de la Institución Educativa el Planchón. Se hizo un análisis del $\mathrm{PEI}, \mathrm{y}$ los proyectos que se vienen adelantando en lo referente a las tematicas relacionadas con la catedra de estudios afro, haciendo una comparación entre los componentes, formas de enseñar y los elementos que fortalecen la identidad cultural en los estudiantes. Estos resultaron permitieron hacer una reestructuración curricular, con la participación de los estudiantes, padres de familia, y comunidades afro del municipio de Puerto Escondido, los cuales se muestran en el desarrollo del presente artículo, finalmente, se describen las estrategias pedagógicas implementadas por parte de los docentes con la restructuración del currículo, aspecto que demuestra los alcances y aportes logrados con el proyecto

\section{Bases Conceptuales}

En Colombia, el interés por los estudios relacionados con el tema de la Etnoeducación viene tomando gran relevancia en el contexto académico, planteándose nuevos referentes metodológicos, pedagógicos y didácticos, que se insertan al amplio debate que se suscita a nivel nacional en las universidades e instituciones educativas.

Se aprecia entonces, un inmenso campo para la identificación, formulación, ejecución de experiencias y propuestas investigativas en temas étnicos, socioeducativos, etnoeducativos y afrocolombianidad, que propicien a nivel educativo el diseño de estrategias de enseñanza que contribuyan a la reflexión de los contextos locales y su interacción con la cultura. La revisión exhaustiva de todo lo publicado, se fundamenta en conocer la bibliografía existente, en algunos casos pueden ser trabajos bastante largos, muy especializados y en otros casos no ofrecen información precisa que se requiere, de allí que se deba hacer un amplio barrido de las fuentes.

Hay que tener presente, que la compresión teórica y metodológica del tema de investigación plantea la necesidad de contar con un análisis minucioso de como se ha venido estudiando este problema, para brindar una orientación clara sobre los avances, tendencias, problemas, vacíos y fortalezas en cada uno de los trabajos y definir el carácter de innovación del presente trabajo con relación a los resultados consultados.

Con el objetivo de facilitar la compresión y análisis del tema, se hace un amplio reconocimiento de los trabajos de investigación que se vienen adelantando por parte de la comunidad científica internacional, nacional y local, él permite tener una visión y reflexión sobre los avances, teorías, enfoques y metodologías que se vienen abordando por diferentes autores. Este barrido, permitió definir una serie de categorías que reflejan los criterios que más sobresalen en los diferentes autores consultados. Aquí se definen las principales categorías de análisis, entre las que se resaltan: la etnoeducación, estrategias pedagógicas y el currículo. 


\section{Categoría: Etnoeducación}

En Colombia existen referente importantes sobre el tema de la etnoeducación, como lo demuestran los trabajos sobre diversidad cultural, multiculturalidad y etnias, reconocimiento de las costumbres, culturas, rituales y las propias cosmovisiones que tienen los diferentes grupos étnicos del pueblo colombiano. Ello proporciona una serie de aspectos o dimensiones que se pueden estudiar y analizar desde el currículo en la Institución Educativa, convirtiéndose ésta en una categoría esencial en la comprensión de la realidad socio educativa de Puerto Escondido.

Se debe aclarar que la etnoeducación es un discurso y una práctica que se vuelve compleja a la hora de ser abordada y estudiada desde el ámbito local, infiere reconocer y entender a través del espacio y el tiempo una serie de relaciones sociales, políticas y económicas, en un contexto nacional, regional, local y en otros casos a nivel global. Algunos autores y los lineamientos expuestos en la catedra de estudios afrocolombianos, sostienen que la etnoeducación tiene presente elementos asociados a lo territorial, intercultural y las características étnicas propias, en este sentido Therán Palacio (2013), expone "La diversidad étnica y cultural se ha convertido en un desafío para la educación colombiana en la medida en que las iniciativas gubernamentales no se han cristalizado en una mejora de las condiciones de vida de los grupos étnicos minoritarios, como lo son los indígenas, los afrodescendientes, y los raizales, entre otros"(2013: 361).

El abordaje de este tema reviste gran importancia, en virtud del reconocimiento que se ha dado a la diversidad pluricultural y étnica, a partir de la Constitución Política de 1991, Ley General de Educación, decretándose la obligatoriedad de la implementación de la etnoeducación en las diferentes instituciones educativas, para el reconocimiento de los plenos derechos, respeto y tolerancia frente a las costumbres y culturas de los pueblos. Pero en la realidad la etnoeducación dentro de los procesos educativos se encuentra como una visión simple y humanista a modo de ideología democrática, no trata de entenderla y abordarla para adaptarla pedagógicamente a las características culturales de los niños, aspecto que involucra el compromiso de los docentes en el logro de este propósito o finalidad de la educación en Colombia.

De otra parte, la UNESCO (2001) plantea como uno de sus objetivos en la Declaración Universal sobre la Educación, la trasmisión de la diversidad cultural para responder a los nuevos desafíos que enfrentan unas sociedades cada vez más plurales. Ello implica, una revisión general del currículo y de los métodos de enseñanza, capacitación y comunicación dentro de los sistemas educativos que hayan sido concebidos para promover la construcción de la identidad nacional. Sigue anotándose que cada área de dicho sistema (formación docente, currículo y libros de texto, métodos de enseñanza y estrategias), debería reconsiderarse desde una perspectiva más amplia, poniendo de relieve los aportes de la diversidad cultural.

Desde este trabajo de investigación, se analiza la importancia que representan los saberes que los estudiantes traen desde su entorno familiar y social, reconociéndose además que ese conocimiento que se encuentra intrínseco en ellos, les da su identidad y los proyecta como seres fortalecidos y 
generadores de desarrollo dentro de su comunidad. De acuerdo a De Sausa (2012), desde las instituciones educativas se deben contribuir con el fortalecimiento de la identidad cultural de una comunidad que poco a poco ha ido siendo permeado por elementos culturales foráneos que más que fortalecerla la han debilitado.

Este planteamiento guarda coherencia con los propósitos que tuvo la investigación, en el sentido de transformar los procesos educativos teniendo presente el entorno y contexto de los estudiantes de las comunidades afrocolombianas y fortalecer los valores culturales y los aportes de está al municipio de Puerto Escondido, puesto que se ha evidenciad una gran descontextualización del currículo de la institución con relación a uno de los grupos étnicos que conforman esta comunidad educativa "Ios afrodescendientes".

\section{Categoría: Estrategias Pedagógicas}

La revisión bibliográfica relacionada con la inclusión de los estudios afro en los procesos formativos de los escolares, evidencian que se ha reconocido en algunos países de América Latina que la multiculturalidad es un tema te interés a nivel internacional, y desde el Programa de Naciones Unidas para el Desarrollo PNUD, se han diseñado programas desarrollo para "Población afrodescendiente de América Latina", y el impulso de políticas públicas para la inclusión de la población afrodescendiente (PNUD 2011), este proceso permite conocer más a fondo quienes somos, de dónde venimos.

En el texto Pedagogía de la alteridad, una dialógica del encuentro con el otro, (Villegas, 2002), se sustenta que las estrategias metodológicas para una educación etnoeducativa afrocolombiana, se desarrolla a través de un proceso de construcción colectiva y participativa en los diferentes escenarios partiendo de la articulación del proceso formativo con la realidad del contexto donde se desarrolle y con las demandas sociales. Estos factores implican un giro epistemológico, ético y pedagógico que afecta la escuela como tradicionalmente se conoce, interviniendo diversos aspectos del quehacer educativo como son: el currículo, la gestión escolar, la formación docente, la intervención didáctica, la evaluación, en otros. En este sentido, la escuela debe ser coherente con la cultura, los valores y las necesidades de los miembros de la comunidad que la conforman respondiendo a una construcción social e histórica de cada pueblo.

Se considera de interés para los grupos étnicos, organizaciones sociales y entes que se dedican a promover la diversidad, la multicultural y multidiplinariedad, analizar y contextualizar los currículos y procesos académicos, para generar la capacidad en los niños de leer el entorno, comprender y aprender teniendo en cuenta las necesidades y las demandas de su contexto, es decir asumir una posición crítica frente a su realidad, de acuerdo a ello, se debe buscar: "Una educación humanizadora y, por tanto, liberadora, exige tomar en serio los puntos fuertes, conocimiento, experiencias, estrategias y valores" (Freire, 2006).

Según Flórez Ochoa (1999: XIX) “La tarea de la pedagogía es identificar y proponer aquellas experiencias y caminos alternos que propician procesos de 
aprendizaje y formación efectivos y placenteros para el desarrollo en armonía con el entorno natural y social de los individuos y de la comunidad". Por lo tanto, desde el aula se debe entender que la cultura enseña a los niños qué pensar y cómo hacerlo, los niños adquieren sus conocimientos ideas, actitudes y valores a partir de su trato con los demás, o aprenden de la exploración solitaria del mundo, sino al apropiarse o "tomar para sí" las formas de actuar y pensar en su cultura, aspecto que orienta los procesos de formación hacia la etnoeducación y en especial la diversidad cultural.

\section{Categoría: Currículo}

En Colombia, han predominado procesos educativos con modelos y currículos inapropiados que se planean desde las altas esferas del Estado, sin la más mínima comprensión de los contextos socioculturales de las instituciones, están desfasados de las singularidades que ellos experimentan y exigen unas bases de construcción nada adecuados para las condiciones actuales sin coherencia con la vida y la realidad institucional. En este sentido, los currículos inclusivos permiten hablar de la interculturalidad como: un proceso de convivencia humana basado en el respeto a la relación recíproca de valores entre varias culturas (Hanoun, 1992 cit Lluch 2003).

Un principio del método Freire citado por Quintero (2007), muy aceptado en las prácticas escolares, consiste en asegurar que todo proceso educativo debe partir de la realidad donde vive el educando. La observación participante de los educadores en un contexto cultural concreto es necesaria para comunicarse con el pueblo. Pensar la realidad y actuar sobre ella es la mejor manera de relacionar teoría y práctica. "La esencia de este método apunta hacia la transformación de un mundo más humano, en un ambiente de amor y esperanza, unión inquebrantable entre acción-reflexión y reconocimiento de sus valores." (Quintero, et. al 2007).

Se hace imprescindible, buscar las estrategias para lograr articular al educando con toda su carga de saberes culturales y sociales con la escuela y sus saberes científicos y académicos. En este sentido Doria (2010), afirma que se entiende la formación como un proceso muy amplio el individuo se forma a través de su relación consigo mismo, con las cosas y con otros individuos (identidad). Por eso cuando se aborda, desde la pedagogía, la reflexión sobre el currículo, se piensa en el tipo de ser humano que se pretende formar, de acuerdo con unas circunstancias históricas y de desarrollo específicas y concretas.

El currículo es proyecto, es intención de formación, podría decirse el espacio en donde se concreta la función de la educación, en tanto muestra los caminos que están por recorrerse. La curricularización es precisamente poner en acción esa intención de formación para hallarles sentido y significado a las acciones humanas a partir del acto educativo mismo. En el texto Pedagogía del conocimiento de Flórez Ochoa (2005) se expone el currículo por procesos, es esencialmente abierto y permeable a la influencia socio-histórica y cultural de la comunidad y del país en el que se inscribe el programa educativo. Anotándose que "la comunidad de la que hacen parte los alumnos es la que 
debiera a partir de la conciencia de sus propios problemas de supervivencia, de convivencia y proyección al futuro, suministrar los ejes temáticos principales que tendrían que formularse en el diseño curricular. Bajo la forma de preguntas que asumirían y procesarían los estudiantes y el profesor no solo del saber universal de las ciencias sino también desde: "el saber global vivo y activo de la tradición cultural de la misma comunidad” (Flórez, 2005: 287).

\section{Metodología}

Este trabajo se basa en el enfoque cualitativo, centrado esencialmente en comprender e interpretar la forma en que se desarrollan los procesos educativos al interior de la Institución Educativa El Planchón. A partir de esto, se buscó estudiar la realidad socioeducativa en su contexto natural, tal y como sucede, intentando sacar sentido e interpretar, los fenómenos de acuerdo con los significados que tienen para las personas implicadas. Según lo expuesto por Rodríguez (1996), este tipo de investigación implica la utilización y recogida de una gran variedad de materiales, como son la entrevista, también las experiencias personales, las historias de vida, las observaciones, los textos históricos, y por último, imágenes y sonidos (p. 39).

La ejecución de este trabajo se basa en el método de Investigación-Acción Participativa (IAP), que es un modelo diferente del tradicional de hacer investigación científica, y que parte del conocimiento de la opinión de las personas que en él participan, considerándolas importantes dentro del proceso de investigación. (Bernal, 2006).

Para tal efecto, se organizaron reuniones con diferentes actores de la comunidad, entre ellos, representantes de las comunidades afrocolombianas del municipio de Puerto Escondido, docentes, estudiantes y padres de familia de la Institución Educativa el Planchón, en la cual se consultó quienes estaban de acuerdo en vincularse en la conformación del grupo IAP. Para tal efecto, decidieron participar 7 docentes de un total de 10 (de las áreas de ciencias naturales, ciencias sociales, inglés, español, ética y valores 2 docentes de básica primaria y la rectora). La institución cuenta 576 estudiantes y se tomó una muestra de 80 estudiantes de los grados 7 y 8, 10, quienes querían aportar al proceso por su formación y conocimientos del tema; por último ,se vincularon al grupo, 5 padres de familia y un representante de la asociación afro del municipio.

Con relación, a los procesos de reflexión, planeación y transformación de la comunidad educativa en los procesos pedagógicos etnoeducativos, se desarrollaron tres fases, las cuales fueron: comprensión del problema a partir de un diagnóstico desde diferentes opiniones de actores que integran la comunidad están los estudiantes, padres de familia, docentes y comunidad afro; apropiación conceptual sobre los aportes histórico-cultural, ancestral de las comunidad afrocolombiana; y construcción de estrategias pedagógicas que fomenten la etnoeducación afrocolombiana en la comunidad educativa el planchón. Cada una de estas fases comprende los siguientes momentos: planear la acción en 
conjunto; ejecutar y observar individual y colectivamente; reflexionar los resultados de las acciones emprendidas; y por último, modificar las prácticas de enseñanza a la luz de los resultados.

En la figura 1, se presenta el diseño esquemático de la investigación acción participativa que se está desarrollando en la institución educativa.

Figura 1. Proceso de investigación acción de Whitehead (1991), propuso el siguiente esquema que apunta a graficar un ciclo de acción que se sostiene en el trabajo participativo.

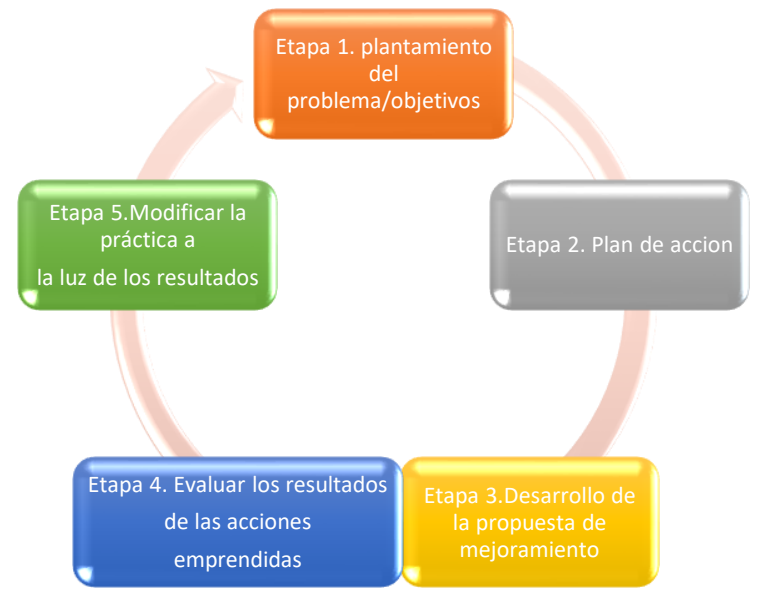

Fuente: Adaptación a partir de Whitehead (1991).

\section{Resultados}

De acuerdo con cada una de las etapas que se propusieron desde el diseño metodológicos, se especifican los alcances y resultados obtenidos en el marco de este estudio:

\section{Fase 1: El Diagnostico del contexto socio-cultural.}

La escuela sin "contenidos culturales" es una ficción, una propuesta vacía, irreal y descontextualizada. el currículo es la expresión y concreción del plan cultural que la institución escolar hace realidad dentro de unas determinadas condiciones que matizan ese proyecto"

José Gimeno Sacristán

Esta fase inició a partir de encuentros con estudiantes, docentes, padres de familia y comunidades afrocolombianas del municipio de Puerto Escondido, con el fin de dialogar sobre los intereses que motivan a la comunidad. En la conversación y reflexión sobre los diferentes problemas que aqueja a la población, se encontró que uno de ellos, es la falta de reconocimiento de las instituciones educativas sobres las características socio- culturales del contexto. A partir de esto, se tomó la decisión de conformar un grupo base que promueva procesos permanentes de construcción de conocimiento fundamentado en la creatividad y el diálogo. En este caso, se pasó de una actitud observadora y diagnostica del 
contexto, a otra más interesada y preocupada por colaborar en el fortalecimiento de los procesos socio culturales en la comunidad de la Institución Educativa el Planchón.

A partir de una visión analítica y crítica del problema que aqueja a la comunidad, se tomó la decisión de la constituir un grupo IAP, que lo integraron representantes de todos los entes. Este grupo, comenzó a reflexionar sobre la interacción que tiene la institución educativa con el contexto socio cultural del Municipio de Puerto Escondido. Todo esto fue sistematizado, haciendo uso de técnicas de recolección de información tales como los grupos de discusión y entrevista en profundidad. Para realizar un diagnóstico de este estudio, se analizó la estructura curricular de la institución Educativa el Planchón y como esta ha venido articulando los procesos de enseñanza aprendizaje de acuerdo con las características del contexto.

Otro elemento importante, fue la discusión y participación del grupo IAP mediante la socialización de los resultados y reflexiones en torno al problema previamente identificado, es decir las causas y consecuencias. Planteándose de esta forma, los objetivos que orientaron algunas actividades que permitieron superar las dificultades a partir de la reflexión sobre la relación del contexto con la estructura curricular. El desarrollo del trabajo de investigación acción ha contemplado la búsqueda de información continua y sistemática, para ello, se diseñaron unos instrumentos que permitieron estudiar y analizar las categorías identificadas en el problema objeto de estudio. Los datos recopilados en los grupos de discusión, entrevistas semi- estructurada y entrevistas en profundidad, se sistematizaron en el programa Atlas-ti versión 7. De este proceso, se obtuvieron unos resultados que se exponen a través de categorías que facilitan el estudio del tema.

La primera categoría de análisis fue relacionada con la estructura del currículo, por lo cual, de los resultados derivados de la compresión de las respuestas emitidas por los actores y miembros del grupo IAP, se resaltan algunos por ejemplo:

Estudiantes "La verdad es que no seño porque aquí los profesores vienen de afuera y solo se basan en lo que les han enseñado y no toman en cuenta las raíces que nosotros tenemos acá" "nos ayudaría a que todos los días esta cultura se inculque en cada uno de nosotros"

"Ni los profesores, ni los estudiantes ni la rectora tenemos ese sentir para inculcarle a cada uno de los estudiantes la cultura afro"

"Las clases se realizan actividades expositivas que te ayudan a desarrollar tus habilidades de expresarte en la sociedad y entorno que te rodea"

"Los contenidos y metodologías con la realidad socio- educativa de nuestro entorno es que por medio de esos contenidos sabemos el origen de todo aquello que nos rodea y conforma nuestro entorno “

\footnotetext{
${ }^{1}$ Análisis por parte del grupo de investigación IAP.
} 

escuela.

\section{Assensus}

Docentes "Los contenidos y metodologías implementadas y desarrolladas en la institución están desarticulada de la realidad que vive nuestro contexto". Creemos que nuestro currículo no está articulado o direccionada a dar solución a la realidad socio- cultural de nuestros educandos y comunidad educativa en general para preparar personas para otros contextos, la cual resulta en un problema a la hora de enfrentarlo a su realidad"

"El currículo no está articulado a la realidad del municipio por lo tanto la institución debe implementar estrategias para que el currículo este contextualizado"

"El currículo debe estar contextualizado de tal forma que los estudiantes conozcan y aprendan su origen, necesidades y solucionar sus problemas" "En la institución no existe un documento donde este manifestado dichos elementos étnicos-educativos

Padres de "Que las clases no solo fueran en el salón sino si no que fueran a excursiones, familia mostrarle la escultura de Bolivia, que los saquen a la playa a ver los corales todo eso me parece espectacular"

"En sociales y en cívica los niños han aprendido el himno o las banderas y cosas de aquí de Puerto"

Comunidades "El recate de la parte cultural del municipio, sobre todo la parte ancestral, como afro es la cultura de nuestras comunidades y la manera de vivir, que no quede en el pasado revivirla y que estos estudiantes conozcan sus raíces y conozcan cuál es su historia. su propia historia"

"Estamos interesados que el municipio de Puerto Escondido todas las instituciones estén focalizadas afro, puesto que en el censo de 1994 aparece que Puerto Escondido tiene una población del 94\% totalmente afro y se le presentó a la alcaldía, una propuesta para que caracterice a la población" "Promover los derechos humanos, sociales, económicos, culturales, ambientales y políticos de las comunidades negras de Puerto Escondido desde una perspectiva étnica, dentro del marco de la diversidad etno-cultural que caracteriza a nuestro país, propiciando cambios de comportamientos proclives a la búsqueda de una identidad cultural; procurando construir una conceptualización filosófica de un pensamiento propio de las etnias afrocolombianas."

Lo anterior, derivó en una reflexión crítica por parte del grupo IAP, permitiendo tomar conciencia acerca de las necesidades de interrogar y someter a debate, de manera colectiva los resultados de un primer diagnóstico desde la óptica de los diferentes entes que integran a la comunidad. Los resultados evidencian un currículo elaborado sin tener presente las características socioculturales de la población. Además, la revisión documental y las entrevistas a docentes de la Institución Educativa El Planchón, permitió reconocer que el plan de estudios sólo tiene en cuenta contenidos, sin objetivos de aprendizaje que expresen su relación con los problemas del contexto. Por otra parte, el modelo pedagógico implantado por los docentes es el tradicional y no tiene relación con el escrito en el PEI (social constructivista). Esto se relaciona con lo que expresan los estudiantes y padres de familia, al considerar que los docentes no relacionan el contexto con los contenidos, porque no son de la región, y además sustentan que las clases se desarrollan de manera expositiva, y no se realizan salidas de campo o eventos que posibiliten la interacción con la comunidad. 
Un aspecto que se debe resaltar es la opinión de las comunidades afro, quienes expresan su preocupación, en el sentido de que las instituciones educativas del municipio de Puerto Escondido, no reconocen que están inmersas en una población donde el 95\% es Afrocolombiana (Plan de Desarrollo, 2012 2015), además proponen que éstas, deben asumir un mayor compromiso social, en especial, para rescatar y fortalecer la identidad cultural.

Con relación a la estructura curricular, se encontraron opiniones que se expresan en torno a que existe una supuesta desarticulación entre la Institución Educativa El Planchón y el contexto, en especial, con su realidad socio educativa. Esto motivó que desde el grupo IAP se redireccionaran los procesos de acuerdo con lo expuesto por Zabalza (1985), en el sentido de que: "es el momento en que la escuela vuelva sobre el territorio en el que actúan de forma tal que se mitigue la perdida acelerada de la propia historia y todos aquellos elementos que nos dotan de identidad (tradiciones, cultura, estilos de vida, sabiduría local)". Surge entonces, la necesidad de plantear y aplicar estrategias de enseñanza que permitan relacionar la identidad cultural de un pueblo, con los saberes de la escuela y desarrollar así una educación contextualizada. En este mismo sentido, la UNESCO (1997) señala que: "la educación tiene una doble misión: enseñar la diversidad de la especie humana y contribuir a una toma de conciencia de las semejanzas y la interdependencia entre todos los seres humanos". por tanto, se requiere una enseñanza que muestre la riqueza de la diversidad para poder valorar, a su vez, la unidad y la solidaridad humana.

Otro elemento analizado en el trabajo de campo fue la caracterización y descripción de los procesos de etnoeducación desde el aula, siendo éste, uno de los ejes fundamentales del proceso de investigación que se desarrolló y que permitió integrar los miembros de la comunidad en un grupo IAP. Algunos apartes de sus voces se presentan a continuación:

"La cultura en mi sangre este yo escucho un bullerengue y eso me hace sentir viva, vengo de una familia donde mis ancestros llevan la cultura de puerto escondido en la sangre y eso me lo han inculcado y esa cultura siempre la llevo presente"

"Si tengo una raíz afro porque soy nacido y criado en puerto escondido y mi familia y mis ancestros también y en la sangre llevo parte afrocolombiana".

"Yo si me siento afrocolombiano porque eso es algo que se siente y se lleva por dentro y que se vive, la

verdad es que cuando escuchamos cualquier tipo o estilo de baile que se interpreta, que sale que se vive, que se brota por los poros entonces, yo me siento afrodescendiente por esa parte “

"El mar que para la temporada trae mucho turista entonces ellos se van en su corazón un sitio que muy bonito, puerto escondido es porque antes llegaban muchos barcos y con ellos gente importante, entonces se llevan esa imagen muy especial de lo que ha sido puerto escondido, yo de mi parte quiero que siempre se siga llevando a cabo y que mejore" "Cultura bullerenguera, la cual llevamos en nuestras venas, nuestras familias y nuestros ancestros siempre nos han inculcado esto una y otra vez para que nosotros lo hagamos" 
Docentes

Padres de familia

Comunidades afro

"Es necesario conocer a fondo el lugar, el contexto, todo lo que uno va a trabajar, para poder desarrollar, para un mejor trabajo"

"El rescate de los valores, el rescate de la cultura de la comunidad donde estamos trabajando"

"Conozco solo algunos apartes de la historia de Puesto Escondido de manera oral por parte de algunos integrantes de la comunidad"

"El currículo debe estar contextualizado de tal forma que los estudiantes conozcan y aprendan su origen, necesidades y solución a sus problemas"

"Los afro, esto esta evidenciado somos negros s costaneros son negros y amo el bullerengue"

"La cultura del bullerengue, los cultivos, las fiestas del plátano"

\begin{abstract}
"La catedra afrocolombiana que tiene que ver con todo lo que son las políticas públicas de etnoeducación"

"La implementación de la etnoeducación ya que la etnoeducación viene de la trietnia que hay en Colombia y en todas las instituciones encontramos diferentes etnias y desde ahí podemos trabajar todo lo que tiene que ver con los valores, con la cultura, con la localidad ancestral y también la implementación de la catedra afro ya que hay mucho material sobre eso, la catedra ayuda a que los estudiantes y profesores se identifiquen y aprendan a querer este proceso y a querer lo que uno es porque nadie quiere lo que no conoce".

"La etnoeducación se preocupa es por la necesidad, por la problemática de la comunidad, por esto debe ser un proceso comunitario donde interactúe la familia, el estudiante y el docente, y esta es la razón por la cual este proceso se ha dado muy lento ya que solo lo llevan los docentes involucrados en el, mas no el resto de los docentes ni directivos, aunque hoy ya se ve un poco de interés de parte de ellos".
\end{abstract}

Posteriormente se pasó a interpretar las expresiones de la comunidad sobre la percepción que se tiene del contexto afrocolombiano en el municipio de Puerto Escondido, en esta fase fue activa la participación de los estudiantes, padres de familia y comunidades afro, quienes resaltaban el conjunto de imágenes que construyen en su contexto social y ellos como asumen sobre sí mismos, un conjunto de costumbres, valores que los identifica. Aspecto que se puede asociar con lo propuesto por el Observatorio Colombiano del Caribe (2010): “..una sociedad que no es capaz de pensarse a sí misma, de generar conocimiento sobre sí misma, de apropiarse del conocimiento sobre sí misma, de evaluarse a sí misma, está llamada a desaparecer". Se aprecia de esta forma que las comunidades afro resaltan la importancia de desarrollar procesos etnoeducativos para mitigar las problemáticas de las comunidades.

Los docentes de la institución asumen que no tienen conocimiento del contexto socio cultural del municipio, pero aceptan que es necesario integrar en sus prácticas pedagógicas el contexto, visto como una herramienta de apoyo que contribuya a fortalecer en los estudiantes los valores, sus creencias anécdotas e historias. La etnoeducación y en especial la Cátedra de Estudios Afro, se convierte entonces, en una estrategia viable y válida que permite a los grupos étnicos identificar y desarrollar propuestas de educación que respondan a sus intereses, necesidades y aspiraciones, de acuerdo con sus características culturales, 
económicas, sociopolíticas, lingüísticas, etc. en una dimensión de articulación intercultural.

Todos estos aportes sobre la problemática que tiene la comunidad educativa, permitieron determinar y delimitar la pregunta de investigación: ¿De qué forma el currículo de la Institución educativa el Planchón articula sus contenidos con su contexto socio-cultural?. De acuerdo con los lineamientos de la investigación acción se concertaron los objetivos utilizando la técnica del árbol del problema (ver tabla 1).

Tabla 1. árbol del problema, organizado por el grupo IAP.

\begin{tabular}{|c|c|}
\hline $\begin{array}{l}\text { Problema central } \\
\text { ¿De qué forma el currículo } \\
\text { de la Institución educativa } \\
\text { el Planchón se articula al } \\
\text { currículo los procesos } \\
\text { etnoeducativos? }\end{array}$ & $\begin{array}{l}\text { Objetivo General } \\
\text { Establecer como la estructura curricular articula los procesos } \\
\text { etnoeducativos al contexto socio cultural de la Institución } \\
\text { Educativa el Planchón - Puerto Escondido. }\end{array}$ \\
\hline $\begin{array}{cl}\text { Causa } & \\
- & \text { Falta de } \\
\text { planeación de las } \\
\text { actividades en la } \\
\text { que se tenga en } \\
\text { cuenta el contexto } \\
\text { - Implementación } \\
\text { de metodología } \\
\text { tradicionales } \\
\text { - Los planes de } \\
\text { área no } \\
\text { responden a las } \\
\text { necesidades } \\
\text { socio- culturales } \\
\text { de los estudiantes }\end{array}$ & $\begin{array}{l}\text { Objetivos Específicos } \\
\text { Analizar la estructura curricular de la institución Educativa, } \\
\text { evidenciando la forma en que se planifican y organizan los } \\
\text { procesos socio educativos. } \\
\text { Describir los procesos pedagógicos y didácticos que utilizan } \\
\text { los docentes para evidenciar la contextualización de los } \\
\text { contenidos curriculares desde el aula. } \\
\text { Diseñar e implementar estrategias pedagógicas que articulen } \\
\text { los procesos etnoeducativo afrocolombianos en el currículo } \\
\text { de la Institución Educativa el Planchón. }\end{array}$ \\
\hline $\begin{array}{l}\text { Consecuencias } \\
\text { - Los estudiantes } \\
\text { se les dificulta } \\
\text { relacionar } \\
\text { conceptos } \\
\text { científicos con su } \\
\text { realidad }\end{array}$ & $\begin{array}{l}\text { Resultados Esperados } \\
\text { - Transformación curricular y pedagógica que articule } \\
\text { los contenidos al contexto socio cultural. } \\
\text { - Los alumnos relacionen los contenidos académicos } \\
\text { con los problemas del contexto. } \\
\text { Incorporar a la escuela con la identidad cultural del } \\
\text { alumnado y con ello consolidar la identidad cultural } \\
\text { de la región. }\end{array}$ \\
\hline
\end{tabular}

Esta fase se basó en una metodología participativa que favoreció la comunicación y la cooperación a través de un trabajo colaborativo, el intercambio de opiniones y expectativas en la construcción curricular. Como estrategia para la evaluación, análisis y empoderamiento conceptual, encaminado a la construcción de un currículo pertinente, flexible y contextualizado se realizaron, entre otras las siguientes técnicas de trabajo como son: exposición temática, grupos de discusión, entrevistas a profundidad y relatorías. Este proceso de reflexión continua, estuvo motivado por preguntas a todos los participantes de la comunidad educativa para diagnosticar y evaluar la pertinencia del currículo, propiciando actividades curriculares abiertas y participativas que servirán para mejorar la sostenibilidad social y cultural de la región; en el grupo de discusión, se 
expuso la necesidad de reorganizar un currículo con identidad y participación de todos los grupos interesados que posibilitó la inclusión de los diferentes entes de la comunidad educativa.

\section{Fase 2. Apropiación de teorías para reflexionar sobre el currículo de la Institución Educativa El Planchón.}

"el currículo no es un concepto, sino una construcción cultural. Esto es, no se trata de un concepto abstracto que tenga algún tipo de exigencia fuera y previamente a la experiencia humana. Más bien es un modo de organización una serie de prácticas educativas" Grundy

En esta etapa, se ha establecieron las bases para el planteamiento de alternativas o propuestas que permitieran el diseño de un plan de acción con la participación y consenso de todos los actores. En este sentido, se puede establecer que la investigación acción participativa, requiere de la realización de procesos de una forma continua y sistemática, que visione la construcción y aplicación de escenarios posibles que tienda a mejorar la situación existente. Las reflexiones iniciales realizadas en el momento de la interpretación y análisis, giraron en torno a la construcción de procesos que transformen el pensamiento social y cultural en la institución educativa desde el currículo, permitiendo a los miembros del grupo IAP, evidenciar en los resultados del diagnóstico, una falta de identidad y reconocimiento de valores relacionados con la cultura afrocolombiana

El propósito esencial de esta etapa estaba orientado a resolver el interrogante ¿Cuáles son las estrategias pedagógicas que se pueden implementar desde el currículo para articular los procesos etnoeducación afrocolombianos en la Institución Educativa el Planchón?. En el análisis realizado a las propuestas expuestas por los docentes, estudiantes y comunidades afro, se llegó al consenso de construir estrategias pedagógicas pensadas desde un contexto sociocultural que incorpore las diferentes propuestas de participación, relacionadas con tres elementos que la constituyen: En primer lugar: tener presente el componente de las representación culturales asociado a la voz de los sujetos, en segundo lugar el componte pedagógico, vinculado con el currículo y por último, una resignificación de su identidad.

Estudiantes "Traigan a alguien nativo de Puerto Escondido y nos oriente más sobre la base de todas las raíces que tenemos aquí en puerto escondido y que nos diga y a ustedes los profesores porque como no son de aquí, entonces nosotros ya tenemos en la mente lo que era antes puerto escondido"

“Obras teatrales para que

podamos expresarnos de una manera más libre, a través de las razas étnicas de nuestros ancestros o podríamos hacerlo en bailes culturales estas actividades se pueden hacer dentro de la misma aula de clases para así nosotros fortalecernos y estaríamos viendo lo que queremos ver sobre la cultura de nuestro pueblo"

"Sacar un grupo de estudiantes de cada salón para que tomen clases sobre los afros y ellos asi esta cultura se las enseñe a los demás compañeros".

"Desarrollar actividades como un concurso de hacer trenzas y bailes de los grupos afro" 
Docentes familia

Comunidades afro

"Bitácora sobre la historia y herencia cultural de la región, Juegos de rol sobre la historia de la comunidad".

"Yo pienso que lo lograríamos con una hora de lectura, que incluiría escritura, para que los estudiantes escriban todo lo que ellos saben sobre la comunidad, sobre la historia, la cultura los valores, todo eso, que consulten con los abuelos, con los bisabuelos, con los padres esa sería la parte escrita, la parte de lectura tratar de conseguir libros y lo mismo que los estudiantes escriban..."

"El rescate de los mitos, leyendas y cuentos de la región para realizar traducción estos textos desde su área de formación al inglés"

"El juego de roles sobre la historia de la comunidad"

"El rescate de la parte cultural del municipio, sobre todo ancestrales, como es la cultura de nuestras comunidades y la manera de vivir, que no queden en el pasado sino revivirlas y que estos estudiantes conozcan sus raíces y conozcan cuál es su historia su propia historia"

\begin{abstract}
"Actividades transversales en todas áreas “
"Actividades lúdicas y recreativas que ayuden a promover los objetivos que se buscan, como combatir la desigualdad"

"Se utilizan las leyendas, cuentos, historias de la región, esto es contado por los niños que a su vez las recibieron de sus padres, y se resaltan las costumbres culturales ancestrales, a veces se invita a alguien de la comunidad para que les hable de su cultura, esto hace que el estudiante se interese por lo propio y en muchos casos termina escribiendo un cuento".

"La historia de los afros en su mayoría no está escrita por eso nosotros debemos contársela, aunque en muchos casos los negros han hecho grandes aportes tecnológicos eso no está escrito, por ejemplo, en la construcción de las pirámides egipcias, en la construcción de las murallas de Cartagena aquí hubo un gran aporte de los negros. Todo esto para que se sientan orgullosos y se identifiquen"
\end{abstract}

El primer aspecto relacionado con las representaciones culturales está caracterizado por la demanda que tienen los actores, por incorporar su mundo simbólico al aprendizaje. El segundo componente para la construcción de la estrategia, es el pedagógico, el cual les permite sistematizar conocimientos de las historias, generar un trabajo en equipo y colaborativo, comunicar la información a toda la comunidad, y articular las áreas del conocimiento para contribuir a la resignificación de las comunidades afro desde las escuelas, es decir, garantizar la contextualización de la educación de acuerdo con la realidad socio cultural del municipio.

De acuerdo con esto, fue pertinente implementar como prueba piloto, una estrategia conocida como el diario de campo, la cual permitió a través de acciones como la observación, el análisis de la realidad y la sistematización de las experiencias en el área de ciencias naturales, consolidar las competencias básicas como lo son el saber, saber hacer y el ser; generándose con ello aprendizaje significativo. Los procesos de seguimiento que se hicieron a esta estrategia se recogieron a través de una entrevista en profundidad a estudiantes participantes: 
Entorno al debate sobre la contextualización curricular se generó una actitud crítica y deliberativa, y se planteó la necesidad de formular una propuesta curricular que aborden los conocimientos, capacidades y valores para educar en un contexto donde los educando reconozca su propia identidad cultural como construcción particular.

Esta fase se basó en una metodología participativa que favoreció la comunicación y la cooperación a través de un trabajo colaborativo, el intercambio de opiniones y expectativas en la construcción curricular. Como estrategia para la evaluación, análisis y empoderamiento conceptual, encaminado a la construcción de un currículo pertinente, flexible y contextualizado se realizaron, entre otras las siguientes técnicas de trabajo como son: exposición temática, grupos de discusión, entrevistas a profundidad y relatorías.

Este proceso de reflexión continua estuvo motivado por preguntas a todos los participantes de la comunidad educativa para diagnosticar y evaluar la pertinencia del currículo, propiciando actividades curriculares abiertas y participativas que servirán para mejorar la sostenibilidad social y cultural de la región. En el grupo de discusión se evidenció la necesidad de reorganizar un currículo con identidad y participación de todos los grupos interesados que posibilitó la inclusión de los diferentes entes de la comunidad educativa.

Fase 3. Reestructuración curricular.

Para la reestructuración curricular en la institución educativa, se tomaron algunas decisiones las cuales fueron plasmada en el Plan de Mejoramiento Institucional (PMI) 2015- 2025. Para esto, se tuvo en cuenta un examen introspectivo del currículo actual, además una visión hacia el exterior analizando lo que otros están haciendo a nivel local, nacional e internacional. De otra parte, se procedió hacer una mirada hacia adelante, es decir lo que hay que hacer para preparar a los jóvenes de la Institución Educativa el Planchón para el futuro, para lo cual, se organizaron unos talleres de contextualización. 
El grupo IAP realizó entrevistas y una revisión documental sobre las comunidades afro para apropiarse del contexto socio cultural de la región para llegar a conocer y entender de forma clara los principales hechos socio históricos, sociales, religiosos y culturales del municipio de Puerto Escondido.

En el abordaje de fuentes bibliográficas sobre el tema el grupo IAP, diseñó una propuesta de reestructuración curricular abierta y participativa teniendo en cuenta los siguientes elementos:

- Reconocimiento de la cultura afrocolombiana como parte importante en la construcción del conocimiento, valorando su propia forma de pensar para incluirlo dentro del currículo para que responda a las características, necesidades aspiraciones e intereses culturales, que le permita proyectarse con identidad hacia otro grupo humano.

- El currículo, se encamina a buscar respuesta pedagógica a los problemas y expectativa de la comunidad y grupo afrocolombianos, y alrededor de ellos se articulan los contenidos y saberes, a través de la opinión de todos los grupos de interesados estudiantes, docentes, directivos, padres de familia y comunidades de afrocolombianas, puesto que esto posibilita que el currículo sea inclusivo y práctico además satisfaga las necesidades de los estudiantes a la que pretende formar.

- Toda cultura, tiene un concepto de su imagen y de hombre, además una sabiduría del mundo que debe proyectarse en la visión y misión de la institución educativa.

- La articulación de la investigación a la malla curricular; a través de preguntas sobre el contexto, permite abordar el conocimiento del entorno e intervenir en el tratamiento de situaciones problémica aportando a la reconstrucción y recate del saber ancestrales de las comunidades afrocolombianas del municipio.

- Plan de clases organizado por secuencias didácticas incorporando las etapas del proceso investigativo.

La propuesta que se desarrolló partió de analizar la importancia de lograr una integración instrumental mediante la cual se organizaron y se le diera sentido a los proyectos pedagógicos, los proyectos institucionales y una serie de componentes tales como: propósitos, metas, disciplinas, recursos y perfiles, para direccionar los fines de la enseñanza y aprendizaje de los estudiantes de acuerdo al modelo pedagógico, a las características culturales y a las necesidades sociales del Municipio de Puerto Escondido. 


\section{Assensus}

\section{Conclusiones}

Uno de los propósitos de la investigación acción participativa gira entorno a la construcción de procesos que transformen el pensamiento social y cultural en la institución educativa. Un hecho de importancia que se puede rescatar con la realización de este trabajo, es incentivar la innovación en los procesos educativos como camino hacia la búsqueda de la calidad. El desarrollo educativo no debe estar desarticulado de la realidad social, ambiental, cultural de su entorno y la construcción de una sociedad justa democrática y participativa debe partir de las expectativas y necesidades circunscritas alrededor de la escuela.

La etnoeducación y en especial la Cátedra de Estudios Afro, se convierte entonces, en una estrategia viable y válida que permite a los grupos étnicos identificar y desarrollar propuestas de educación que respondan a sus intereses, necesidades y aspiraciones, de acuerdo con sus características culturales, económicas, sociopolíticas, lingüísticas, etc. en una dimensión de articulación intercultural.

La innovación de las prácticas educativas, que promuevan la exploración de preguntas problémicas infiere conocer los conceptos y enfoques que permiten aplicar el aprendizaje significativo, colaborativo y situado. Estas le brindaron a los docentes nuevas opciones para cambiar el modelo o paradigma que se aplicaba en la Institución Educativa el Planchón, desarrollando proyectos de aula, en las áreas de las Ciencias Naturales, Ciencias Sociales, Español, Inglés y Artística. 


\section{Referencias Bibliográficas}

Álvarez M. G (2010), Diseñar el currículo universitario: un proceso de suma complejidad.

De Sousa, B. (2010). Refundación del estado en América Latina: perspectiva desde una epistemología del sur: México, D.F: Siglo XXI Editores

Doria Rudy. (2014) El Currículo: eje de la gestión escolar para la formación y el desarrollo humano.

Flórez Ochoa, Rafael. (2005). Pedagogía del conocimiento, 28 Bogotá: Mac Graw Hill

Hernández, S (2007). El constructivismo social como apoyo en el aprendizaje en línea, 46-62. Disponible http://www.redalyc.org/pdf/688/68800705.pdf. Consulta 15 de mayo 2014

http://cmc.inmc.us/papers/cmc2004-290.pdf Consulta 13 de septiembre 2014.

Lago de Zota, A, (2012, 29 de mayo). Educación para ciudadanos del mundo con identidad afrodescendiente: caso institución educativa Antonia Santos, Cartagena de Indias, Colombia. Historia de la educación latinoamericana Volumen (14), p. 54

Latorre Antonio (2007), La investigación-acción: Conocer y cambiar la práctica educativa, España: Graò.

Reinoso H. (2012), Caracterización del proceso de contextualización curricular para la enseñanza de la química: un estudio de caso

Rodríguez, Hilda Mar y Echeverri, J. (2004). Práctica y diario pedagógico. (La estructura de la memoria. Narrar-se). Alternativas, 35, 67-74.

Rioseco, M \& Romero, R. (Septiembre, 1997). La contextualización de la enseñanza como elemento facilitador del aprendizaje significativo, Moreira (coord.), encuentro internacional sobre el aprendizaje significativo, España

Torres, María..(2004). Identidad cultura y sujeto político. El discurso "negado" en la identidad puntana. Revista Alternativas, 131-148

UNESCO:http://www.brookings.edu/ /media/Research/Files/Reports/2013/02/learning\%20m etrics/LMTF_report1_exec_sum_

UNESCO: http://unesdoc.unesco.org/images/0012/001246/124687e.pdf\#page=72

Zabalza, Miguel. (2012). Territorio, cultura y contextualización curricular. Revista Journal (8), p.22 